\title{
Ontology Assisted Query Reformulation Using the Semantic and Assertion Capabilities of OWL-DL Ontologies
}

\author{
Kamran Munir \\ University of the West of England \\ Centre for Complex Coop. Systems \\ BS16 1QY, Bristol, UK \\ +44-1173-283279 \\ Kamran.Munir@cern.ch
}

\author{
Mohammed Odeh \\ University of the West of England \\ Centre for Complex Coop. Systems \\ BS16 1QY, Bristol, UK \\ $+44-1173-283700$ \\ Mohammed.Odeh@uwe.ac.uk
}

\author{
Richard McClatchey \\ University of the West of England \\ Centre for Complex Coop. Systems \\ BS16 1QY, Bristol, UK \\ +44-1173-283176
}

\begin{abstract}
End users of recent biomedical information systems are often unaware of the storage structure and access mechanisms of the underlying data sources and can require simplified mechanisms for writing domain specific complex queries. This research aims to assist users and their applications in formulating queries without requiring complete knowledge of the information structure of the underlying data sources. To achieve this, query reformulation techniques and algorithms have been developed that can interpret ontology-based search criteria and associated domain knowledge in order to reformulate a relational query. These query reformulation algorithms exploit the semantic relationships and assertion capabilities of OWL-DL based domain ontologies. In this paper, this approach is applied to the integrated database schema of the EU funded Health-e-Child $(\mathrm{HeC})$ project with the aim of providing ontology assisted query reformulation techniques to simplify the global access that is needed to millions of medical records across the UK and Europe.
\end{abstract}

\section{INTRODUCTION}

\subsection{The Problem in General and Motivation}

Information technology today has been widely adopted in modern medical practice, especially in the support of data management. However little has been achieved in the use of computational techniques to exploit integrated medical information in research. In recent years, there has been a substantial increase in the volume and complexity of data and knowledge available to the medical research community. To enable the use of this knowledge in clinical studies, users generally require an integrated view of medical data across a number of data sources [1]. Clinicians, who are mostly the end users of medical data analysis systems, are normally unaware of the storage structure and access mechanisms of the underlying data sources. Consequently, they require simplified mechanisms for generating queries.

The Health-e-Child (HeC) project [2] aims to develop an integrated platform for European paediatrics, enabling data integration between spatially distributed clinicians and bringing together information produced in different departments or

Permission to make digital or hard copies of all or part of this work for personal or classroom use is granted without fee provided that copies are not made or distributed for profit or commercial advantage and that copies bear this notice and the full citation on the first page. To copy otherwise, to republish, to post on servers or to redistribute to lists, requires prior specific permission and/or a fee.

IDEAS08 2008, September 10-12, Coimbra [Portugal]

Copyright (C2008 ACM 978-1-60558-188-0/08/09 \$5.00 multiple hospitals. The emphasis of the $\mathrm{HeC}$ data integration process is on providing "universality of information". Its cornerstone is the integration of information across biomedical abstractions, whereby all layers of biomedical information can be 'vertically integrated' [3] (i.e. integration across cellular, organ, disease, patient and population data). The approach advocated in this paper surrounds the provision of access to an $\mathrm{HeC}$ Integrated Data Model [4] plus semantics-driven and transparent query services using manually developed description logic based ontologies. In this regard a framework has been previously presented in [5] which provides transparent query services to access the data.

\subsection{HeC Query Reformulation Services}

The work presented in this paper exploits the semantic relationships and assertion capabilities of an OWL-DL based ontology in order to capture the domain knowledge and to provide query formulation and reformulation services to the clinicians and their medical applications. To this end a query reformulation system has been developed as middleware between the client applications and distributed data sources (as shown in figure 1). This query reformulation system parses the query and interprets the meaning of the end-user's query terms. In the case where the client request is not automatically resolved or the end-user does not really know what he/she is looking for (or how to ask for available information), the system allows him/her to see and interpret such information. Both of these features enable the construction of a meaningful query.

The process of parsing and interpreting the meaning of the query terms is enabled by the use of source metadata information and domain knowledge that is defined in terms of ontological concepts. These concepts are classified within the internal structure of the ontology. The ontological information is then used for situation-based information querying.

\subsection{Query Reformulation and Ontologies}

An ontology generally represents a shared, agreed and detailed model (or set of concepts) of a problem domain. One advantage in the use of ontologies in the $\mathrm{HeC}$ system is their ability to resolve any semantic heterogeneities that are present within the data. Ontologies can define links between different types of semantic knowledge. Hence, they can aid particularly the resolution of terms for queries and other general search strategies, thus improving the search results that are presented to clinicians. The facts that ontologies are machine-processable and human understandable are especially useful in this regard [6]. 


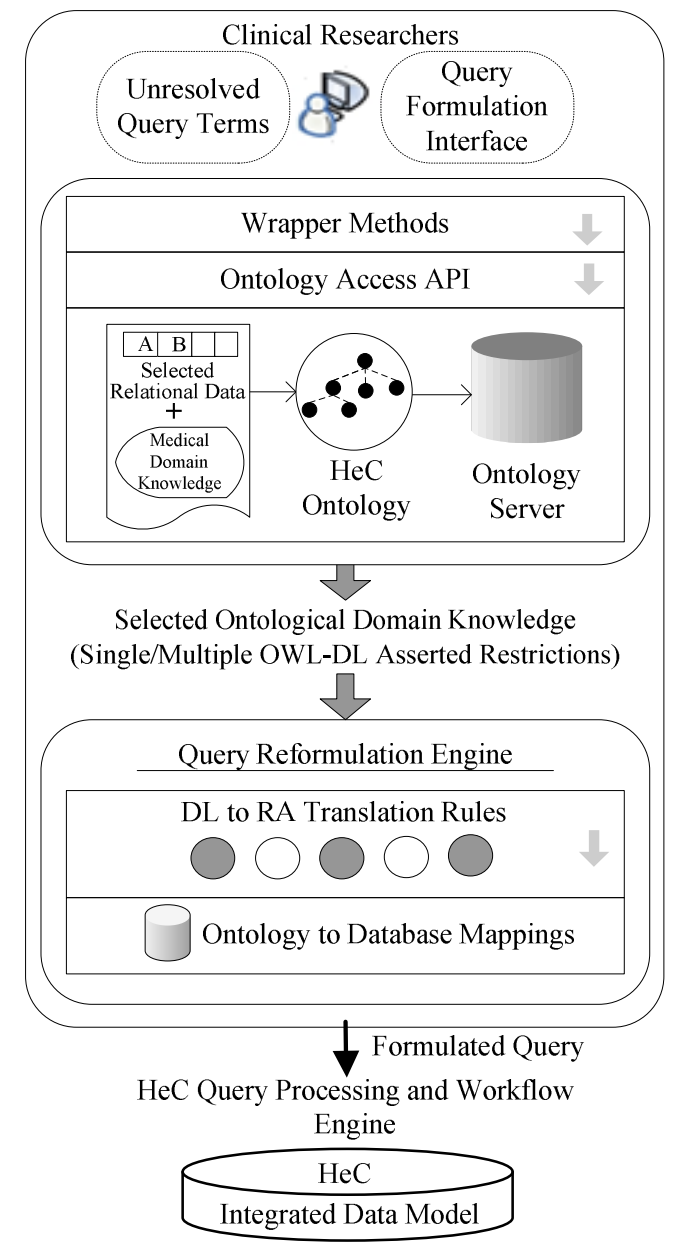

\section{Figure 1. Query Reformulation System Architecture in Health-e-Child}

There are many biomedical ontologies in existence although few, if any, that support query reformulation. The example below explains how ontologies can be used in formulating a query. Due to space limitations, it is not practical to describe the complete $\mathrm{HeC}$ database scheme. Thus, as a running example in this paper, we use the following small subset of the database relations from our Patients' database, the complete $\mathrm{HeC}$ integrated data model can be found in [4].

(1) patients_data (patient id, clinical test name, clinical_ test_value, description, ...)

(2) clinical_test (clinical_test_id, clinical_test_name, ...)

(3) clinical_test_values (id, clinical_test_id, ct_value,...)

(4) patient (․ㅏ, description, ...)

The rows in the table clinical_test store all the possible clinical tests that can be taken for a particular patient. The clinical_test_values table stores all the possible clinical test results for any particular clinical test. The patients_data table references the patients, their clinical tests and results of medical tests.

As mentioned previously, ontologies can aid in the area of query reformulation. One example is when a query is reformulated according to the $\mathrm{HeC}$ ontology with the concept of 'Astrocytoma Tumor' (a form of tumor in the brain). The user may enter a query into the system stating "Give me all MRI scan images of brains for children with an Astrocytoma Tumor disease in a specific age group". This query cannot be fully resolved by the $\mathrm{HeC}$ data model because there is no direct information available in the databases that matches with the term 'Astrocytoma Tumor'. Here the query reformulation system receives a simple input into the system as 'Astrocytoma Tumor', the system then extracts all of the clinical tests and related values that confirms the possibility of Astrocytoma Tumor disease in the brain.

The system uses the power of the HeC ontology to determine that clinical test results for example orthopedic_sequelea with values severe symptomatic and life threatening are the related clinical tests for Astrocytoma Tumor. Hence, the system not only returns the result as a set of related clinical tests but also returns the respective reformulated query to access this information according to the underlying data model. Such requirements which require resolution of terms from the query reformulation system can occur frequently. Examples of queries with similar requirements include: (1) when a clinician wants to study a particular disease (2) when a clinician wants to study patients who are suspected of having a particular disease and (3) when a clinician wants to compare a patient's medical data with patients who have a particular disease, and within a specific age group.

The subset of the above mentioned $\mathrm{HeC}$ patients' database does not contain information that is directly related to some of the above mentioned terms in queries. Therefore, in order to retrieve the desired query results in the absence of a query reformulation system, clinicians are normally required to perform all of the following operations:

1. To describe the clinical tests that are related to the study of particular diseases;

2. To describe clinical tests and associated results that need to be "true" for one particular patient to be selected as a suspect for particular diseases;

3. To understand how clinical test data is stored in the integrated $\mathrm{HeC}$ database; and

4. To write complex SQL queries to select the desired information.

A user may want to write a more complex query by involving any number of comparisons using union, intersection, equivalence or negation operations. Current developments in the field of ontology languages allow ontologies to be more expressive when ontological information is used to formulate complex queries. To achieve this, generic query reformulation techniques have been developed that interpret ontological knowledge to reformulate queries or to assist the users in formulating their queries.

The remainder of this paper is organised as follows. Section 2 presents related work in this area. Section 3 introduces the Query Reformulation system architecture. This section also focuses on the ontological representation for reformulating queries, and discusses the ontology-relational translations that provide (relational) query reformulation services. Finally, Section 4 concludes the paper and considers the current status of research and directions for future work.

\section{RELATED WORK}

Currently, there are several tools available that can transform relational databases into ontologies. DataGenie [7] is a plug-in for Protégé [8] that imports data from a relational database to an 
ontology. Similarly, related work has been carried out by [9], [10] and [11] on the transformations between relational databases and ontologies. These mappings are fairly trivial: each table maps to a class, each column to a data type property and each row to an instance. In addition, the foreign key columns are used to link an instance of a class to instances of another class. In this research, while using domain ontologies to reformulate relational queries, some of the basic rules to generate domain ontology from relational schema remain the same. However, our relational schema to ontology transformation is different in the sense that 'all' relational data are not transformed and then stored as ontology instances.

The work presented in [12] supports the specialisation or generalisation of the base or filler concepts to build database specific queries interactively. However, that querying facility cannot generally be applied to queries where the corresponding data is not available as ontology instances in the respective data sources. Therefore, implementing such a querying facility in the situation where there are legacy data sources would require significant changes to the database schema. For example, such legacy data sources could involve the medical records of patients (as in $\mathrm{HeC}$ ). In these cases it is likely to be difficult to manipulate the database schema because of the huge database volume, the associated security protocols and the legacy applications that access them. However, in our approach other than the metadata information no data is stored as ontology instances or directly linked to ontological concepts. Therefore, no manipulation of the data at the schema level is required. The database metadata is defined within the domain ontology without limiting user queries to the specialisation or generalisation of ontological concepts.

Some of the existing ontology-based information retrieval approaches use RDF [12], [13], [14] and [15] structures which, although yielding schema information, provide insufficient knowledge for query reformulation. These approaches also lack the details of what needs to be included in the ontology from the data sources along with the domain knowledge to drive the process of query reformulation. The focus of these approaches (for example [15]) remains towards interactive query generation through nondirected graphs supporting multiple natural languages. Furthermore, considerable work has been carried out by [16] in addressing the problem of data integration and the interoperation of heterogeneous XML sources using an ontology-based framework, where a global ontology is generated and expressed in an RDF Schema (RDFS) [17]. This system depends on an ontology to define the set of terms that can be used in a query. To query data, users need to be familiar with the overall terms and relationships in the ontology. This can sometimes be problematic for users who do not fully understand the system and thus face difficulty while navigating through large ontologies to select the appropriate terms.

Unlike the approaches in [18], [19], [20], [21] and [22] our system does not store all data from a data-source as part of the ontology or link it directly with ontology concepts. Often it is not practically feasible to store all data as part of a certain domain ontology especially for systems with large amounts of data. The data that is stored as part of the ontology needs to be loaded in memory to perform Select query operations. Most of these approaches have used RDF as an ontology development language. However RDF is too weak to describe resources in sufficient detail since it lacks localised range and domain constraints and there is no support for cardinality constraints. In the current research OWL-DL is the ontology development language that is used to specify the concepts with related assertions that drive the process of query reformulation, since it has greater support for expressing semantics when compared to RDF and RDFS. Work has been carried out in [23] to map a domain ontology to a domain conceptual data model. In this research several mapping rules have been proposed that guide the transformation from domain ontology to conceptual schema. One of these mapping rules describes the transformation of ontology properties to entities-attributes in the conceptual model. In this paper, this rule has been extended to define mappings between an OWL ontology to a data source schema.

A database relational schema provides a logical map of the information content of the database along with related semantic data control assertions, following the relational model. On the other hand, although ontology schemas share the conceptualisation aspects of relational schemas, the ontology model specifications and, in particular OWL ontologies, (used in this research) are based on Description Logic theory [24] and are referred to as OWL-DL. In order to represent a relational data model in OWL-DL, respective transformations of the relational model to DL remain a critical requirement in order to achieve consistency and completeness of these transformations. In relation to this, work has been carried out in [25] which describes the relationship between entities in the entity-relationship (ER) model and DL theory. In the current research some of the basic ontology to DL transformation rules are employed and extended to handle the requirements for reformulating database queries.

\section{THE QUERY REFORMULATION SYSTEM}

The query reformulation system reported in this paper has two major subsystems: (1) the Ontology Knowledge Interface and (2) the Query Reformulation Engine. The Ontology Knowledge Interface subsystem is composed of three components: (a) an ontology creation process to assist in reformulating queries, (b) an ontology server, and (c) an ontology assisted query reformulation process. The Query Reformulation Engine is composed of (a) query reformulation algorithms and (b) ontology to data source mappings.

\subsection{The Ontological Knowledge Interface}

As a first step towards ontology assisted query reformulation, an OWL-DL ontology is created which stores database metadata information within the basic ontology structure. In order to support the re-use, maintainability and evolution of the ontology, a traditional iterative process is adapted for ontology engineering consisting of ontology modeling and ontology validation. In this regard, the metadata from the $\mathrm{HeC}$ integrated data model is mapped into disjoint independent trees which are recombined into an ontology using definitions and axioms to represent the relationships in an explicit fashion.

The main elements of a relational database include relations (tables), columns, and constraints (assertions). Equivalently, the ontological model includes classes, properties, assertions and other semantics. However, for the purpose of query reformulation our approach does not require the domain ontology to include all constructs of the relational model. The domain knowledge is expressed in terms of ontology property assertions that need to be 
consistent with the basic ontology structure. It is also possible to include the domain knowledge from widely available domain ontologies. The mapping rules were developed and have been presented in [5]; that paper explains what needs to be included in the ontology to support the query reformulation process.

In our system either the client applications or the user (through an interactive GUI) interacts with the ontology knowledge interface layer to describe the query terms that cannot be automatically resolved from the data sources. The ontology knowledge interface provides access to ontological concepts classified within the internal structure of the ontology.

\subsubsection{Ontological Representation}

This section explains how a subset of the patients' database metadata, used to drive the process of generating queries, is represented in an OWL-DL ontology. Both the domain knowledge and the metadata of the $\mathrm{HeC}$ data model are stored in the ontology; a small subset of this metadata is shown here:

$D B$ relation: clinical_tests $(P K:$ ct_id)

\begin{tabular}{|l|l|}
\hline ct_id & name \\
\hline 1 & Headaches \\
\hline 2 & double_vision \\
\hline 3 & orthopedic_sequelea \\
\hline 4 & bacterial_infection \\
\hline$\ldots$ & $\ldots \ldots \ldots$ \\
\hline
\end{tabular}

$D B$ relation: $c l i n i c a l \_t e s t \_v a l u e s\left(P K: i d, c t \_i d\right)$

\begin{tabular}{|l|l|l|}
\hline id & ct_id & ct_value (classifications) \\
\hline 3 & 2 & True \\
\hline 4 & 2 & False \\
\hline 5 & 3 & moderate symptomatic \\
\hline 6 & 3 & life threatening \\
\hline 7 & 3 & sever symptomatic \\
\hline$\ldots$ & $\ldots$ & $\ldots . . .$. \\
\hline
\end{tabular}

FK: $c t$ id reference clinical_tests(ct_id)

The clinical_test_values table stores the possible clinical test results for any particular clinical test id $\left(c t \_i d\right)$. Here $c t \_i d$ (clinical test id) is referenced using the clinical_test table. Firstly, a clinical_tests class is added to the ontology that contains all of the clinical tests. These would include for example, headache, double_vision, thrombosis_sequelea, orthopaedic_sequelea as subclasses, and containing only one instance for each class. The second class, namely clinical_test_values, has been defined as a (common) parent class to hold all possible clinical test values for each clinical.

Due to the fact that patients' clinical tests can hold diverse result set values for each clinical test the further subclasses of clinical_test_values (e.g. headachesValues, doubleVisionValues, thrombosisSequeleaValues and orthopedicSequeleaValues) have been created. Each of these subclass concepts contains individuals; some of them are shown in Figure 2. We define clinical_tests and clinical_test_values classes as disjoint so that an individual (or object) cannot be an instance of more than one of these two classes.

Secondly, the object properties, namely hasClinicalTestName, and the sub-properties of object property hasClinicalTestValue are added. In order to provide a two-way search capability through query reformulation algorithms, these sub-properties have a corresponding inverse property. If a property links individual 'a' to individual ' $b$ ', then its inverse property links individual ' $b$ ' to individual 'a'. For example, the Clinical Tests individual 'orthopaedic_sequelea' is linked with the individuals: moderate_symptomatic, life_threatening and severe_symptomatic with the property hasOrthopaedicSequeleaValue. But, OWL's inverse property isValueOf links the individuals moderate_symptomatic, life_threatening and severe_symptomatic with the concept orthopaedic_sequelea.

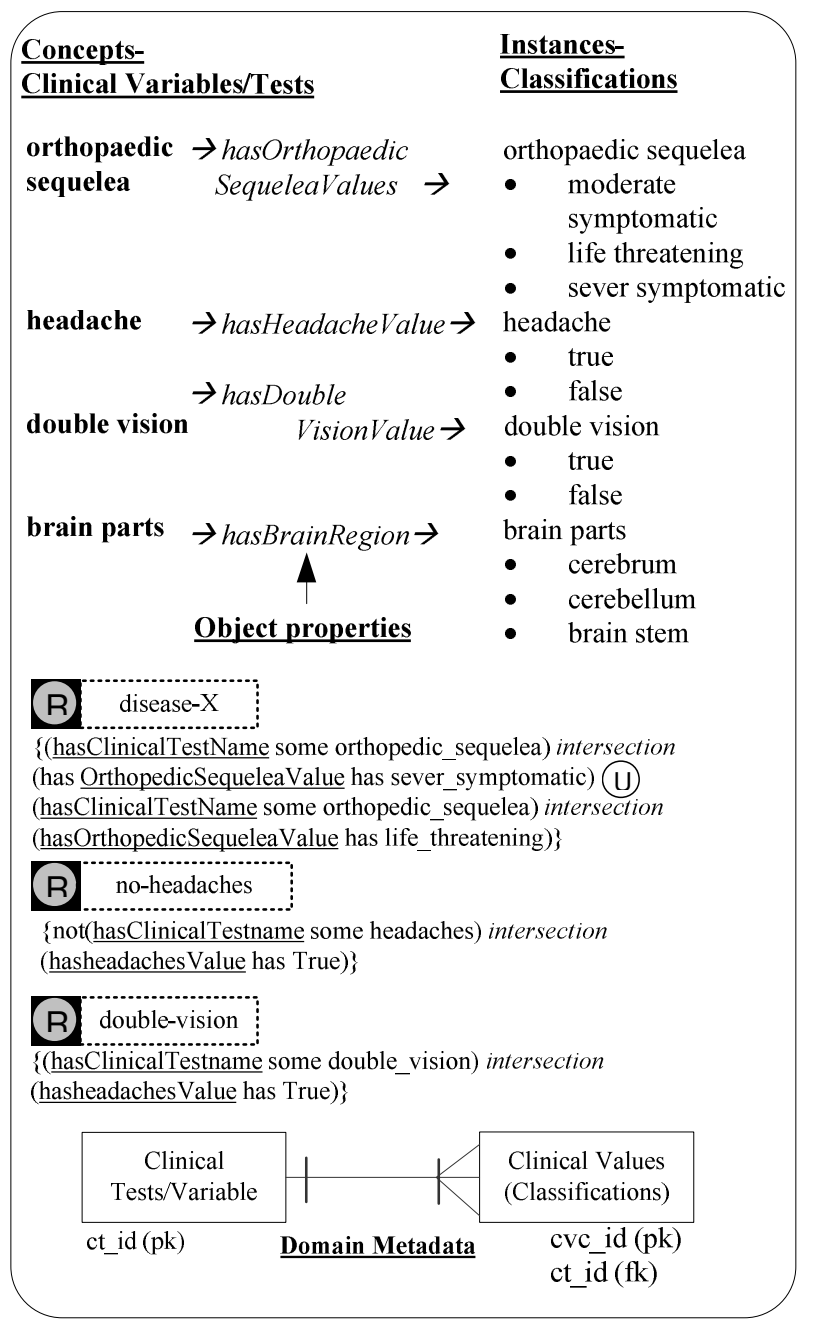

Figure 2. An Example of Ontology Knowledge Representation

If the end users (i.e. clinicians) are accessing the query reformulation system for interactive query generation then during the whole process the users are guided to select the next applicable ontology concept with the corresponding individuals or values. To achieve this task each of the ontology properties has a domain and a range specified. Object properties link individuals from the domain to individuals from the range. For example, the sub-properties of hasClinicalTestValue link individuals belonging to the class Clinical_Tests to individuals belonging to the Clinical_Test_Values class. This is applied to all of the properties available in the ontology, for example the domain of the hasOrthopaedicSequeleaValue property is orthopaedic_sequelea and the range is orthopaedic_sequelea_values as shown (as property links) in Figure 2.

Once the properties with domains and ranges have been defined, then specific class instances are associated with other instances 
using these object properties. These property links are used to capture the user search criteria within the ontology concepts, or independently from the domain ontology if it is to be further utilised by other users.

Once the basic structural elements of the domain ontology have been defined they are further enriched with domain knowledge. The domain knowledge is expressed in terms of OWL-DL property assertions that need to be consistent with the basic ontology structure. We store this domain knowledge as ontology concepts. In this way the consistency of the domain knowledge with ontology concepts is verified using an Ontology Reasoner [26]. Concept restrictions are used to describe conditions for the selection of records that match some given criteria. These restrictions could be either singular or complex involving many conditions. The query reformulation engine uses these restrictions to reformulate queries by translating the DL constructs into relational queries. For the purpose of reformulating queries, the OWL-DL property restrictions are utilised. The ontology concepts describing a particular disease study embody the associated domain knowledge as well as the search criteria.

\subsubsection{The Ontology Server}

Once the ontology has been defined it is then processed and stored in a database. The ontological knowledge interface (as shown in Figure 1) interacts with the ontology server to retrieve the ontological information. This information is then used to assist the users to define their search criteria (if required) and to generate reformulated database queries by receiving unresolved query terms from client applications. The consistency of the domain knowledge with ontology concepts can be verified using an ontology Reasoner (e.g. FaCT++, Racer) [26]; however, if the domain knowledge is to be accessed from a database then this requires the implementation of a consistency check mechanism to ensure coherence with the ontology. This domain knowledge is used by the query reformulation algorithms to reformulate queries conforming to the schema(s) of the underlying data sources.

\subsubsection{The Ontology Assisted Query Formulation Process}

The ontology knowledge interface can receive requests from the client applications as well as from the end users. In situations when end users are directly accessing the ontology knowledge interface, the user could define a new search criterion or select from the existing domain knowledge to formulate a query. The users' search criteria are described using the ontology property restrictions, concepts/sub-concepts and instances. An individual must satisfy all the conditions that are specified as ontology property restrictions to be a member of any named concept. These restrictions could be either singular or complex ones involving many conditions. For example, restrictions are used to define conditions for the selection of relevant patient records that match a given criteria. A GUI interface, the so-called "Ontology Assisted Query Formulation Wizard", is provided for this task which guides the user in defining the search conditions by making full use of the supporting domain ontology.

For example, in order to generate the query (query-1) where a user wants to retrieve clinical data for each patient related to the study of Brain Tumor Disease-X, the selection criterion is described as OWL property assertions, e.g. by using an ontology property namely "hasClinicalTestName". Once defined, the whole search criteria are saved as a new ontological concept for example, brain_tumor_disease_x_study. In this example, it is assumed that the double_vision, headaches and orthopaedic_sequelea are the clinical test names related to brain tumor disease-X. This information is described and saved as follows:

Concept name: brain_tumor_disease_x_study

(OWL expression)

hasClinicalTestName some double_vision union

hasclinicaltestname some headaches union hasclinicaltestname some orthopaedic_sequelea

For the situations where the ontology knowledge interface receives requests from the client applications to reformulate queries for the 'unresolved query terms', the ontology access API accesses the ontology to extract relevant OWL-DL assertions. For example (query-2), when a user wants to retrieve information about patients who are suspected to have a particular Brain Tumor Disease-Y (e.g. 'Astrocytoma Tumor') the system receives the query term 'Astrocytoma Tumor' and extracts all of the clinical tests and related values that confirms the possibility of the Astrocytoma Tumor disease in the brain. The system uses the $\mathrm{HeC}$ ontology to determine that the clinical test results for example orthopaedic_sequelea with values severe_symptomatic and life_threatening are the related clinical tests for Astrocytoma Tumor. These conditions need to be satisfied in order to indicate a suspected case of brain tumor disease $x$ :

Concept name: brain_tumor_disease_y_suspects

OWL expression: \{someValuesRestriction

(hasClinicalTestName some orthopedic_sequelea

Intersection

hasClinicalTestStringValue has severe_symptomatic)

someValuesRestriction

(hasClinicalTestName some orthopedic_sequelea

Intersection

hasClinicalTestStringValue has life_threatening)\}

These query conditions could, of course, be more complex since it could involve multiple ontology assertions using a mixture of union, intersection, equivalence and negation operations even within each property restriction. The query reformulation for such cases involves the handling of all different situations. In the next section, we show how these object property assertions, domain knowledge and associated database mappings are utilised to reformulate the respective query.

\subsection{The Query Reformulation Engine}

The Query Reformulation Engine is composed of query reformulation algorithms and ontology-database mappings. The query reformulation interface passes the extracted relevant ontological information to the query reformulation engine. The query reformulation algorithms interpret and transform the OWL Description Logic constructs into corresponding Relational queries. The 'mappings' table is created automatically during the ontology processing that stores the information about ontology property links, database name, table name, column name, primary and foreign keys. Once created this mapping table only contains the information about ontology properties, which is then updated with the database metadata information.

For example, from the selection conditions defined for query 1 (as discussed in section 3.1.3), it can be deduced that the 
double_vision, headaches and orthopaedic_sequelea are the clinical tests related to Brain Tumor Disease-X Study and defined with the ' $O R$ ' condition. Here the ' $O R$ ' condition for all parts of the test condition implies that there is a 'UNION' operation within each test condition for the data in Patients' database. Finally, the formulated query in this case will retrieve the patient data from the Patient_Information database view/table where patient_information. clinical_test_name matches any of the following values $\{$ double_vision, headaches, orthopaedic_sequelea $\}$. Query 2 is more complex than query 1 and from the previously defined selection conditions for query 2 , we deduce that the following asserted restrictions are indicative of Brain Tumor Disease- $X$ :

Table 1. Ontology asserted restrictions for the Brain Tumor Disease-X suspects

\begin{tabular}{|l|l|l|}
\hline $\begin{array}{l}\text { Ontology } \\
\text { Restrictions }\end{array}$ & Ontology Properties & Test Conditions \\
\hline condition-1A & $\begin{array}{l}\text { hasClinical } \\
\text { TestName }\end{array}$ & $\begin{array}{l}\text { orthopaedic_- } \\
\text { sequelea }\end{array}$ \\
\hline $\begin{array}{l}\text { AND(condition } \\
1 B)\end{array}$ & $\begin{array}{l}\text { hasOrthopedic } \\
\text { SequeleaValue }\end{array}$ & $\begin{array}{l}\text { severe__ } \\
\text { symptomatic }\end{array}$ \\
\hline condition-2A & $\begin{array}{l}\text { hasClinical } \\
\text { TestName }\end{array}$ & $\begin{array}{l}\text { orthopaedic_ } \\
\text { sequelea }\end{array}$ \\
\hline $\begin{array}{l}\text { AND(condition } \\
2 B)\end{array}$ & $\begin{array}{l}\text { hasOrthopedic } \\
\text { SequeleaValue }\end{array}$ & life_threatening \\
\hline
\end{tabular}

Here the clinical test variable orthopaedic_sequelea with the clinical test values 'severe_symptomatic' and 'life_threatening' respectively are defined as restrictions for brain_tumor_disease_x_suspects. In this case the reformulated query for query 2 will retrieve all patients that have all Clinical Tests recorded for Disease-X with specific values for each Clinical Test i.e. orthopaedic_sequelea $=$ 'severe_symptomatic' and orthopaedic_sequelea $=$ 'life_threatening'.

As described previously (in section 3.1) the property hasClinicalTestValue is a parent property of the hasDoubleVisionValue, hasHeadachesValue and hasOrthopaedicSequeleaValue objects. In this approach we require mapping definitions only for the parent properties. The following are the mappings for the ontology properties hasClinicalTestName and hasClinicalTestValue for the Patient_Information database view.

hasClinicalTestName $\rightarrow$ (belongs to) clinical_test_name hasClinicalTestValue $\rightarrow$ (belongs to) clinical_test_value

In the next section, we outline the mappings between an ontology model and a relational database model. These mappings provide us with the ground on which we have based (and implemented) our query reformulation algorithms in the query reformulation engine to handle the possible Description Logic expressions to respective Relational Query translations.

\subsection{Mappings from an Ontology Model to a Relational Model}

A relational data model aims at establishing links between user and domain requirements and describes the logical structure and contents of the data. However, it is often necessary to clarify the meaning of the entities and their properties for a specific domain of interest to aid understanding. An ontology is one way of describing these entities along with their properties in the real world [6]. Recently, semantic web ontology languages have been used to express different types of ontologies and associated languages such as OWL to help in modeling the real world more accurately. These ontologies play a significant role in information system modeling and have the ability to represent the conceptual data models using ontological theories [23]. Moreover (as discussed previously in sections 1.3 and 2), work has also been reported in [10] and in particular the R2O System [11] that describes the mappings between a relational database schema and an ontology.



(a)

One concept in the ontology

having only

one instance maps to one

record in a DB

relation. In this

case the

corresponding

properties map

to each column

and each

instance of a

concept maps

to a column

value.

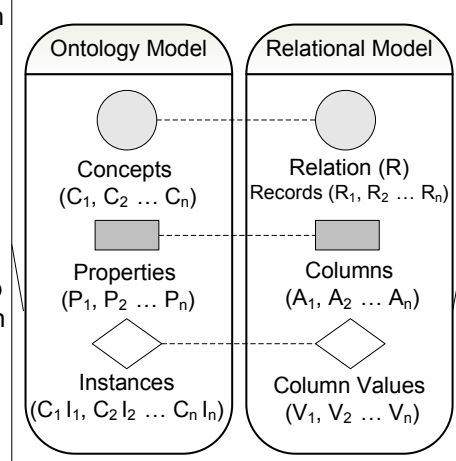

One DB relation instantiates more than one concept in the ontology, but only one instance per concept. In this case columns of the relation maps the properties and each column value to an instance of (b) each concept.

One concept in
the ontology
having multiple
instances map
to one or more
then one
record in a DB
relation. In this
case
corresponding
properties map
to each column
and the
instances of a
concept map
to the column
values.
values.

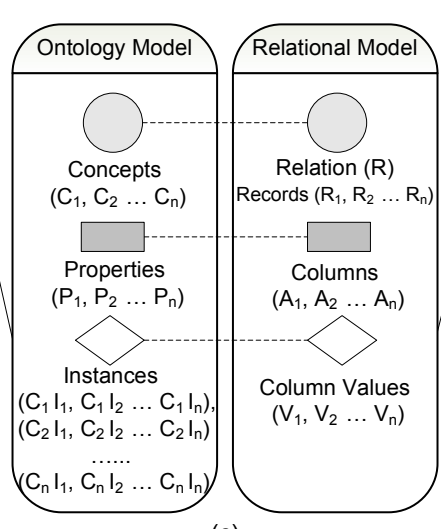

(c)
One DB relation instantiate more than one concept in the ontology, but multiple instances per concept. In this case columns of the relation maps the properties and each column value to one or more instances of each concept.

Figure 3(a, b, c). Mappings between an ontology model and a relational model

Figure 3 presents different mapping situations that arise from ontology-to-relational and relational-to-ontology model mapping scenarios and are covered by our query reformulation algorithms (detailed examples of these mappings are reported in [5]). Here the mappings are expressed as a set of correspondences that relate 
the vocabulary of a relational model (table/relation, column etc) with an ontology model (concept, property etc) and vice versa.

In the relational database paradigm, a logical data model may be accessed through SQL which is based on the Relational Algebra (RA), whereas OWL-DL is based on Description Logic [24]. Therefore, we base our translations on Description Logic and Relational Algebra, to work with any database that implements the SQL standard. In DL, a given DL is defined by a set of concepts and a role forming operator. The smallest set propositionally closest to DL is $A L C$ (Attributive Language with Complements) where the concepts are constructed using Union, Intersection, allValuesFrom, someValuesFrom and complementOf written as $\cup, \cap, \forall, \exists$, and $\neg$, respectively. The 'all' in allValuesFrom is the universal qualifier whereas the 'some' in someValuesFrom is the extensional qualifier. The someValuesFrom (hasClass) and allValuesFrom (toClass) constructs are applied on classes or subclasses while specifying classes and restrictions, whereas the hasValue is used with instances.

In the next section, we outline the DL to Relational Algebra (RA) translation heuristic rules rather than a formal approach to achieve this translation from OWL-DL ontological queries to relational queries that can be executed by the relational query processor of a relational database management system.

\subsection{Translation of OWL DL Constructs into Relational Queries}

From this point onwards, the following conventions (according to the mappings defined in the previous section) have been used in the translation rules from DL to RA queries.

- $\zeta$ represents an ontology or ontology fragment

- $\mathrm{Q}_{\mathrm{R}}$ is the formulated query in RA

- $\mathrm{R}$ is a database relation/view

- $\mathrm{C}$ is the ontology concept/class

- $\mathrm{C}_{1}, \mathrm{C}_{2} \ldots \mathrm{C}_{\mathrm{n}}$ are multiple ontology concepts/classes

- $\mathrm{P}$ is an ontology property (mapped to a database column)

- $\mathrm{P}_{1}, \mathrm{P}_{2} \ldots \mathrm{P}_{\mathrm{n}}$ represent multiple ontology properties (mapped to database columns)

- I is an ontology instance (mapped to a database column-value)

- $\mathrm{I}_{1}, \mathrm{I}_{2} \ldots \mathrm{I}_{\mathrm{n}}$ represent ontology concept-instances (mapped to database column values)

- pk_column is the primary key column for a database relation $\mathrm{R}$

\subsubsection{Translations for the allValuesFrom DL construct}

The allValuesFrom restriction excludes the possibility of further additions for a given property. The 'allValuesFrom' is interpreted as "only", such that saying all values coming from a given class is the same as saying that values may only come from that class. While defining OWL property assertions the "allValuesFrom" may be used in the following ways:

(1) Concept (C) having only one instance (I): $<$ object-property>allValuesFrom(Class)

(2) Concept $(\mathrm{C})$ having multiple instances $\left(\mathrm{I}_{1} \ldots \mathrm{I}_{\mathrm{n}}\right)$ : $<$ object-property>allValuesFrom \{class-instance $<$ space $>$ class-instance ...... \}

For both of these cases the following query reformulation rules are used to generate a relational query.
1. An Ontology assertion with an allValuesFrom $(\forall)$ restriction for a property $(\mathrm{P})$ on a concept $(\mathrm{C})$, implies:

If ' $\mathrm{C} \in \zeta$ I allValuesFrom $(\forall)$ of $\mathrm{P}$ toClass $\mathrm{C}$ ' then the translated relational algebra query will be:

$$
Q_{R}=\sigma_{P_{p k_{-} \text {column }} \neg I N\left(\pi_{p k_{-} \text {column }}\left(\sigma_{P_{<>>C}}(R)\right)\right.}(R)
$$

2. If $\mathrm{C} \in \zeta \mid$ allValuesFrom $(\forall)$ of $\mathrm{P}$ toClass (class-instances) $\left(\mathrm{I}_{1} \mathrm{I}_{2} \ldots . \mathrm{I}_{\mathrm{n}}\right)$ then

(Here $\left\{I_{1}<\right.$ space $>I_{2}<$ space $\left.>\ldots . . I_{n}\right\}$ are the class instances for property $\mathrm{P}$ )

$$
\begin{aligned}
Q_{R}= & \sigma_{P_{p k_{-} \text {column }} \neg I N} \\
& \left(\pi_{p k_{\text {c column }}}\left(\sigma_{\neg\left(P=I_{1} \vee P=I_{2} \vee \ldots . \vee P=I_{n}\right)}(R)\right)(R)\right.
\end{aligned}
$$

\subsubsection{Translations for the someValuesFrom DL construct}

The "someValuesFrom" is interpreted as 'some', such that the values may come from a given class. The DL to RA translation for a single someValuesFrom property restriction on a concept (C), having only one instance (I) is fairly straightforward. For example, in such a case the ontology property maps to the column name and the concept maps to the column value. Therefore, we only present the translations concerned with the more than one occurrence of the someValuesFrom property restriction, and with the Union or Intersection or both operations within each restriction. For such cases the following scenarios (and combinations of all these) can occur:

Scenario 1: Multiple someValuesFrom ( $\exists$ ) (restrictions), with a Union operation within each restriction, and a (similar) property defines each concept.

Such a scenario only occurs for a class having subclasses, and a property defines the parent class as a Range class.

$\left(\left(<\right.\right.$ property $P>$ someValuesFrom $<$ class $\left.C_{l}>\right)$ Union

$\left(<\right.$ property $P>$ someValuesFrom $<$ class $C_{2}>$ )) $>\ldots \ldots$.

$\left(<\right.$ property $P>$ someValuesFrom $<$ class $C_{n}>$

Here, the ontology assertions with the someValuesFrom $(\exists)$ property restrictions for $\left(\mathrm{C}_{1}, \mathrm{C}_{2} \ldots \mathrm{C}_{\mathrm{n}}\right)$ with Union operation within each someValuesFrom property restriction, imply:

If $\mathrm{C} \in \zeta \mathrm{I}\left(\right.$ someValuesFrom $(\exists)$ of $\mathrm{P}$ some $\left.\left(\mathrm{C}_{1}, \mathrm{C}_{2} \ldots \mathrm{C}_{\mathrm{n}}\right)\right)$ then

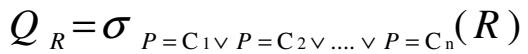

Scenario 2: Multiple someValuesFrom ( $\exists$ ), with a Union operation within each restriction, and a distinct property defines each concept.

$\left(\left(<\right.\right.$ property $P_{1}>$ someValuesFrom $<$ class $\left.C_{1}>\right)$ Union

$\left(<\right.$ property $P_{2}>$ someValuesFrom $<$ class $\left.C_{2}>\right)$ )

If $\mathrm{C} \in \zeta \mathrm{I}$ (someValuesFrom $(\exists)$ of $\mathrm{P}\left(\mathrm{P}_{1} \cup \mathrm{P}_{2} \cup \ldots \cup \mathrm{P}_{\mathrm{n}}\right)$ some $\left(\mathrm{C}_{1}, \mathrm{C}_{2} \ldots \mathrm{C}_{\mathrm{n}}\right)$ then

(Here $\left(\mathrm{P}_{1}, \mathrm{P}_{2} \ldots \mathrm{P}_{\mathrm{n}}\right)$ are the ontology properties for the concepts $\left(\mathrm{C}_{1}, \mathrm{C}_{2} \ldots \mathrm{C}_{\mathrm{n}}\right)$ having only one instance per class.)

$Q_{R}=\sigma_{P_{1}=C_{1} \vee P_{2}=C_{2} \vee \ldots . . \vee P_{n}=C_{n}}(R)$ 
Scenario 3: Multiple someValuesFrom ( $\exists$ ), with an Intersection operation within each restriction, and a distinct property defines each concept.

Such a scenario only occurs for distinct someValuesFrom properties, and a property defines the parent class as a Range class.

$\left(\left(<\right.\right.$ property $P_{1}>$ someValuesFrom $<$ class $\left.C_{l}>\right)$ Intersection $\left(<\right.$ property $P_{2}>$ someValues From $<$ class $C_{2}>$ ))

If $\mathrm{C} \in \zeta \mathrm{I}$ (someValuesFrom $(\exists)$ of $\mathrm{P}\left(\mathrm{P}_{1} \cap \mathrm{P}_{2} \cap \ldots \cap \mathrm{P}_{\mathrm{n}}\right)$ some $\left(\mathrm{C}_{1}, \mathrm{C}_{2} \ldots \mathrm{C}_{\mathrm{n}}\right)$ then

$$
Q_{R}=\sigma_{P_{1}=C_{1} \wedge P_{2}=C_{2} \wedge \ldots . . \wedge P_{n}=C_{n}}(R)
$$

Scenario 4: A someValuesFrom ( $\exists$ ) restriction with the multiple instances of a concept.

Such a scenario can occur when multiple instances of concept are defined with a someValuesFrom property restriction.

$<$ object-property> someValuesFrom \{class-instance $<$ space $>$ class-instance ...... $\}$

If $\mathrm{C} \in \zeta$ I someValuesFrom ( $\exists$ ) of $\mathrm{P}$ hasClass (class-instances)

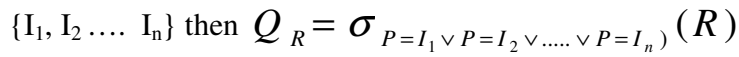

(Here $\left\{I_{1}<\right.$ space $>I_{2}<$ space $\left.>\ldots . . I_{n}\right\}$ are the class instances for property $\mathrm{P}$ )

\subsubsection{Translations for the complementOf $D L$ construct}

The complement Of DL construct selects all individuals that do not fall under the specified restriction(s). For a single ontology assertion with only one complementOf property restriction the translation is trivial. For example, in such a case the ontology property maps to the column name and the individual maps to the column value with a NOT equal-to condition. Therefore, here we only present the translations concerned with the more than one occurrence of the complementOf (hasValues) property restriction, and with the Union or Intersection or both operations within each restriction. For such cases the following three scenarios (and combinations of all these) can occur:

Scenario 1: A complement $O f$ construct, with a Union operation within each hasValue property restriction.

complementof (hasValues of $I_{1}$ Union hasValues of $I_{2}$ Union ..... Union hasValues of $I_{n}$ )

If $\mathrm{C} \in \zeta$ I complementOf (hasValues $(\exists)$ of $\mathrm{P}$ has $\left(\mathrm{I}_{1} \cup \mathrm{I}_{2}\right.$ $\left.\left.\cup \ldots \cup \mathrm{I}_{\mathrm{n}}\right)\right)$ then $Q_{R}=\sigma_{\neg\left(P=I_{1} \vee P=I_{2} \vee \ldots . . \vee P=I_{n}\right)}(R)$

Scenario 2: Multiple complementOf constructs, with an Intersection operation within each restriction.

complement Of (hasValues of $I_{1}$ ) Intersection complementOf (hasValues of $\mathrm{I}_{2}$ ) Intersection ...... Intersection complementOf (hasValues of $I_{n}$ )

If $\mathrm{C} \in \zeta \mid<$ complementOf> hasValues $(\exists)$ of $\mathrm{P}$ has $\left(\mathrm{I}_{1} \cap \mathrm{I}_{2}\right.$ $\left.\bigcap \ldots \cap \mathrm{I}_{\mathrm{n}}\right)$ then

$$
Q_{R}=\sigma_{\neg\left(P=I_{1}\right) \wedge \neg\left(P=I_{2}\right) \wedge \ldots . . \wedge \neg\left(P=I_{n}\right)}(T)
$$

\subsubsection{Translations for the hasValue DL construct}

A hasValue(has) restriction, denoted by the symbol ( $\ni$ ), describes the set of individuals that have at least one relationship along a specified property to a specific individual. Some of the basic translations for hasValue property restrictions are almost similar to the scenarios 1,2 and 3 described previously for the suggested someValuesFrom DL construct translation. The only major difference between them is that hasValue describes the set of individuals and someValuesFrom describes the ontology concept(s). Therefore, in this section we present two further example translations concerned with the more than one occurrence of the hasValues property restriction, with the Union or Intersection or both operations within each property restriction.

Scenario 1: Multiple hasValue constructs, with the Union or Intersection or both operation(s).

$\left(\left(<\right.\right.$ property $P_{l}>$ hasValue <instance $\left.I_{1 i}>\right)$ Union

(<property $P_{1}>$ hasValue <instance $I_{2 i}>$ ) Union......)

Intersection

$\left(<\right.$ property $P_{2}>$ hasValue <instance $\left.I_{I j}>\right) \ldots \ldots$

If $\mathrm{C} \in \zeta \mid$ (hasValues $(\exists)$ of $\mathrm{P}_{1 \mathrm{i}}$ has $\left.\left(\mathrm{I}_{1 \mathrm{i}} \cup \mathrm{I}_{2 \mathrm{i}} \cup \ldots \cup \mathrm{I}_{\mathrm{ni}}\right)\right) \bigcap \mathrm{IU}$

(hasValues $(\exists)$ of $\left(\mathrm{P}_{1 \mathrm{j}} \cap \mathrm{P}_{2 \mathrm{j}} \cap \ldots \cap \mathrm{P}_{\mathrm{nj}}\right)$ has $\left.\left(\mathrm{I}_{1 \mathrm{j}}, \mathrm{I}_{2 \mathrm{j}} \ldots \mathrm{I}_{\mathrm{ni}}\right)\right)$ then

$$
\begin{aligned}
Q_{R}=\sigma_{(} & \\
& \left(P_{1 \mathrm{i}}=I_{1 \mathrm{i}} \vee P_{1 \mathrm{i}}=I_{2 \mathrm{i}} \vee \ldots . . \vee P_{1 \mathrm{i}}=I_{n i}\right) \text { and } / \text { or } \\
& \left(\left(P_{1 \mathrm{j}}=I_{1 \mathrm{j}}\right) \wedge\left(P_{2 \mathrm{j}}=I_{2 \mathrm{j}}\right) \wedge \ldots . . \wedge\left(P_{\mathrm{nj}}=I_{\mathrm{nj}}\right)\right)
\end{aligned}
$$

Scenario 2: Multiple assertions of a hasValue construct.

Such a scenario can occur only with the distinct properties.

$<$ property $P_{1}>$ hasValue(has) $<$ instance $I>$

$<$ property $P_{2}>$ hasValue(has) $<$ instance $I>\ldots . .$.

$<$ property $P_{n}>$ hasValue(has) <instance $I>$

If $\mathrm{C} \in \zeta$ | multiple assertions | (hasValues $(\exists)$ of $\mathrm{P}_{1}$ has I), (hasValues $(\exists)$ of $\mathrm{P}_{2}$ has $\mathrm{I}$ ) ...... (hasValues $(\exists)$ of $\mathrm{P}_{\mathrm{n}}$ has $\mathrm{I}$ ) then $Q_{R}=\sigma_{P_{1}=I \wedge P_{2}=I \wedge \ldots . . \wedge P_{n}=I}(R)$

3.4.5 Translations for the combinations of the someValuesFrom (i.e. hasClass) and the hasValue (i.e. hasInstance) DL constructs

As described previously, the "someValuesFrom" DL construct is interpreted as "some", such that the values may come from a given class and a hasValue restriction describes the set of individuals. In this section, we present the example translations concerning the combinations of both, the someValuesFrom (i.e. hasClass) and the hasValue (i.e. hasInstance) DL constructs. For such cases the following three scenarios (and also the all possible combinations of the previously specified scenarios for the someValuesFrom, the hasValue and the complementOf DL constructs can occur:

For the following examples, $\mathrm{P}_{\mathrm{s}}$ represents the someValuesFrom $(\exists)$ and $\mathrm{P}_{\mathrm{h}}$ represents the hasValue $(\exists)$ related ontology properties.

Scenario 1: A single restriction with the (combination of) someValuesFrom (i.e. hasClass) and the hasValue (i.e. hasInstance) DL constructs. 
The someValuesFrom and hasValue constructs are used together when restrictions are to be placed on the instance(s) (as hasValue) of a particular concept (i.e. hasClass). The following combination can be interpreted as 'value may come from a class ' $C$ ' that matches the instance ' $I$ '. This is particularly useful when a selection condition is to be applied on more then one column of a DB relation/view.

$\left(\left(<\right.\right.$ property $P_{s}>$ someValuesFrom $<$ class $\left.C>\right)$

Intersection (<property $P_{h}>$ hasValue <instance $\left.I>\right)$ )

If $\mathrm{C} \in \zeta \mathrm{I}$ (someValuesFrom $(\exists)$ of $\mathrm{P}_{\mathrm{s}}$ some $\mathrm{C}$ ) $\bigcap$ (hasValue $(\exists)$ of $\mathrm{P}_{\mathrm{h}}$ has I) then $Q_{R}=\sigma_{P_{S}=C \wedge P_{h}=I}(R)$

In such a case the Intersection (And) operation is applied between the hasClass and hasValue constructs, and both of the conditions need to be true for the selection of a particular record.

Scenario 2: Multiple restrictions with the (combination of) someValuesFrom and the hasValue DL constructs, with a Union operation within each combine (someValuesFrom, hasValue) restriction.

In such a scenario the conditions are applied to the multiple concepts and their corresponding instances.

( $<$ property $P_{s}>$ someValuesFrom $<$ class $C_{l}>$

Intersection $<$ property $P_{h}>$ hasValue <instance $I>$ ) Union

( $<$ property $P_{s}>$ someValuesFrom $<$ class $C_{2}>$

Intersection <property $P_{h}>$ hasValue <instance I $>$ )Union ...

If $\mathrm{C} \in \zeta \mathrm{I}\left(\left(\exists\right.\right.$ of $\mathrm{P}_{\mathrm{s}}$ some $\mathrm{C}_{1} \cap \ni$ of $\mathrm{P}_{\mathrm{h}}$ has $\left.\mathrm{I}\right) \mathrm{U}$

( $\exists$ of $\mathrm{P}_{\mathrm{s}}$ some $\mathrm{C}_{2} \cap \ni$ of $\mathrm{P}_{\mathrm{h}}$ has $\mathrm{I}$ ) ....

( $\exists$ of $\mathrm{P}_{\mathrm{s}}$ some $\mathrm{C}_{\mathrm{n}} \cap \exists$ of $\mathrm{P}_{\mathrm{h}}$ has $\mathrm{I}$ )) then

(Here ' $\exists$ ' is represents someValuesFrom and ' $\exists$ ' represents hasValues.)

$$
\begin{aligned}
& Q_{R}=\sigma_{\left(P_{s}=C_{1} \wedge P_{h}=I\right) \vee\left(P_{s}=C_{2} \wedge P_{h}=I\right)} \\
& \vee \ldots . . \vee\left(P_{s}=C_{n} \wedge P_{h}=I\right)
\end{aligned}
$$

As mentioned earlier in this paper, the ontology-to-database mapping information is stored within the ontology server, which includes the information about ontology property links, database name, table names, column names, primary and foreign keys. Once the query reformulation engine transforms the DL constructs into respective relational constructs, the ontology property information is updated with the database information. Finally, the reformulated relational query is passed to the query processing engine for execution.

Although the SQL relational algebra operations cover many cases as specified above, there are situations in which some additional translations are required. For example, matching for different date formats, partial string matching etc.; these are not covered in this paper. Regarding database join operations, we have considered only the natural join operation and have not dealt directly with the theta, semi and outer join operations between the database tables. For these join operations database views have been used to test the translations.

This approach has been applied on a part of the integrated $\mathrm{HeC}$ patients' database schema along with the implementation of a graphical user interface (GUI) to perform query formulation and reformulation tasks. Due to scope and space limitations, detailed GUI descriptions have not been discussed in this paper. The prototype system has been presented to the $\mathrm{HeC}$ consortium and domain experts who have confirmed its potential functionality.

The current work in the project centres around evaluating the correctness of the above translation heuristics applied to a larger data-set and to extend the query reformulation algorithms, where necessary.

\section{CONCLUSIONS}

The central aim of this work was to provide the end users and their applications with query reformulation services using a domain ontology, with the main task of generating relational queries without requiring a complete knowledge of the information structure and access mechanisms of the underlying data sources. This involved the design of a query reformulation architecture with two main layers, the ontological knowledge interface and the query reformulation engine respectively.

The task of query reformulation has been automated by the successive incremental development of algorithms, to test the extent to which this procedure could be effectively automated. One of the key merits of this approach is that no interpretation of data needs to be carried out to be stored as ontology instances. This is clearly beneficial since the interpretation of data in existing data sources may cause serious scalability issues with existing legacy applications. Secondly, it does not require its users to be familiar with the overall contents of the ontology to generate queries. This is helpful for the users who do not fully understand the system; navigating in large ontologies to select appropriate terms can itself be problematic. Moreover, the ontological information is accessed from the ontology server through customized wrapper methods, which is favorable while using large domain ontologies. Furthermore, the query reformulation engine is composed of generic Description Logic to Relational Query translation algorithms, and therefore can be easily employed for other domains.

While the implemented rules to translate OWL-DL queries to respective relational queries are heuristic based, further work is being carried out in the context of the $\mathrm{HeC}$ project to provide a formal ground to translate from description logic based ontologies to relational queries. The latter work will enable us to formally inform the verifiability of these anticipated translations from a point of view of correctness and consistency. Also, there are issues that remain to be handled when using this heuristic approach. This is especially true when establishing the order and combinations of ontological expressions before they can be translated to relational queries.

Despite these limitations, the current research work has provided us with a deeper insight into the problem by formulating a set of heuristics as a step to guiding the anticipated automation of this ontology-relational translation process. Finally, we anticipate that this approach will pave the way for a reflective process where results of queries' execution will enrich the current repository of domain ontologies.

\section{ACKNOWLEDGMENTS}

The authors would like to acknowledge the support of the EU in funding this work and the valuable assistance of all partners in the Health-e-Child project, with special thanks to colleagues working for UWE at CERN, Geneva and colleagues at UWE, Bristol in particular Dr Peter Bloodsworth. 


\section{REFERENCES}

[1] D. Caragea, J. Pathak, J. Bao, A. Silvescu, C. Andorf, D. Dobbs and V. Honavar, "Information Integration from Semantically Heterogeneous Biological Data Sources", DEXA Workshops: Proceedings of the 3rd International Workshop on Biological Data Management, pp. 580-584, IEEE Computer Society, Copenhagen, Denmark, 2005

[2] The Information Societies Technology Project: Health-eChild, EU Contract IST-2004-027749

[3] A. Anjum, P. Bloodsworth, A. Branson, T. Hauer, R. McClatchey, K. Munir, D. Rogulin and J. Shamdasani, "The Requirements for Ontologies in Medical Data Integration: A Case Study", Eleventh International Database Engineering \& Applications Symposium (IDEAS) vol. 6, , pp. 308-314, IEEE Press, Banff, Canada, 2007

[4] A. Branson, T. Hauer, R. McClatchey, D. Rogulin and J. Shamdasani, "A Data Model for Integrating Heterogeneous Medical Data in the Health-e-Child Project", Accepted at the HealthGrid'08 Conference, Chicago USA, 2008

[5] K. Munir, M. Odeh, P. Bloodsworth and R. McClatchey, "Using Assertion Capabilities of an OWL-Based Ontology for Query Formulation", 3rd International Conference on Information \& Communication Technologies: from Theory to Applications (ICTTA), IEEE, Damascus, Syria, 2008

[6] A. Gómez-Pérez and O. Corcho "Ontology Languages for the Semantic Web," IEEE Intelligent Systems vol. 17, no. 1, pp. 54-60, 2002

[7] Z. Xu, S. Zhang and Y. Dong, "Mapping between Relational Database Schema and OWL Ontology for Deep Annotation", Web Intelligence, 2006. WI 2006. IEEE/WIC/ACM International Conference on Web Intelligence, pp. 248-552, ISBN: 0-7695-2747-7, Hong Kong, 2006

[8] "The Protégé Ontology Editor and Knowledge Acquisition System", Access URL: http://protege.stanford.edu , Accessed on: Feb, 2008

[9] N. Konstantinou, D. E. Spanos et.al. , "An Approach to an Intermediate Layer between Ontologies and Relational Database Contents", International Workshop on Web Information Systems Modeling (WISM), 2006

[10] Y. An, A. Borgida and J. Mylopoulos, "Inferring Complex Semantic Mappings between Relational Tables and Ontologies from Simple Correspondences", In Proceedings of On The Move to Meaningful Internet Systems (OTM'05): CoopIS, DOA, and ODBASE, pp. 1152-1169, Springer Verlag, Agia Napa, Cyprus, 2005

[11] J. Barrasa, O. Corcho, G. Shen and A. Gomez-Perez, "R2O, an Extensible and Semantically Based Database-to-ontology Mapping Language", 2nd Workshop on Semantic Web and Databases (SWDB) , 2004

[12] N.W. Paton, R. Stevens, P. Baker, C.A. Goble, S. Bechhofer and A. Brass, "Query Processing in the TAMBIS Bioinformatics Source Integration System", Proceedings of the IEEE International Conference on Scientific and Statistical Databases (SSDBM) , pp. 138-147 , 1999

[13] E. Mena, A. Illarramendi, V. Kashyap and A. Sheth "OBSERVER: An Approach for Query Processing in Global Information Systems based on Interoperation across Pre- existing Ontologies," Journal on Distributed and Parallel Databases vol. 8, no. 2, pp. 223-271, 2000

[14] P.G. D. Baer, E. Kapetanios, S. Keuser , "A Semantics Based Interactive Query Formulation Technique", User Interfaces to Data Intensive Systems: Second International Workshop on User Interfaces to Data Intensive Systems, pp. 43-49, ISBN: 0-7695-0834-0, Zurich, Switzerland, 2001

[15] E. Kapetanios, D. Baer, B. Glaus and P. Groenewoud, "Data Querying and Analysis through Integration of Intentional and Extensional Semantics", 16th International Conference on Scientific and Statistical Database Management (SSDBM) , pp. 353-356 , ISBN: 0-7695-2146-0, 2004

[16] H. Xiao and I. Cruz "Integrating and Exchanging XML Data using Ontologies," Journal on Data Semantics VI: Special Issue on Emergent Semantics vol. 6, pp. 67-89, 2006

[17] D. Brickley and R. Guha ,"RDF Vocabulary Description Language 1.0: RDF Schema", Accessed on: 10-10, 2006

[18] C. B. Necib and J. C. Freytag, "Query Processing using Ontologies",CAiSE, pp. 167-186, Porto, Portugal, 2005

[19] A.L. Rector, S. Bechhofer, C.A. Goble, I. Horrocks, W.A. Nowlan and W.D. Solomon "The GRAIL Concept Modelling Language for Medical Terminology," Artificial Intelligence in Medicine vol. 9, pp. 139-171, 1997

[20] D. Calvanese, G.D. Giacomo, D. Lembo, M. Lenzerini, A. Poggi and R. Rosati , "Ontology-based Database Access", Proc. of the 15th Italian Conf. on Database Systems (SEBD) , pp. 324-331, 2007

[21] D. Calvanese, G.D. Giacomo, D. Lembo, M. Lenzerini, A. Poggi and R. Rosati , "Linking Data to Ontologies: The Description Logic DL-LiteA", In Proc. of the 2nd Workshop on OWL: Experiences and Directions (OWLED) , 2006

[22] Y. Arens, C. A. Knoblock and W. M. Shen "Query Reformulation for Dynamic Information Integration," Journal of Intelligent Information Systems - Special Issue on Intelligent Information Integration vol. 6, no. 2, pp. 99-130, 1996

[23] H. El-Ghalayini, M. Odeh, R. McClatchey and T. Solomonides, "Reverse Engineering Domain Ontologies to Conceptual Data Models", Proceedings of the 23rd IASTED International Conference on Databases and Applications, pp. 222-227, Austria, 2005

[24] F. Baader, I. Horrocks and U. Sattler, "Description Logics as Ontology Languages for the Semantic Web", Mechanizing Mathematical Reasoning: Essays in Honor of Jörg Siekmann, number 2605 in Lecture Notes in Artificial Intelligence, pp. 228-248, Springer, 2005

[25] A. Borgida, M. Lenzerini and R. Rosati, "Description Logics for Databases "The description logic handbook: theory, implementation, and applicationspp. 462-484,.., ISBN: 0521-78176-0, Cambridge University Press , 2003

[26] V. Haarslev and R. Möller, "An OWL Reasoning Agent for the Semantic Web", In Proc. of the International Workshop on Applications, Products and Services of Web-based Support Systems, in conjunction with IEEE/WIC International Conference on Web Intelligence, pp. 91-95, Halifax Canada, 2003 